(2) Open Access Full Text Article

\title{
Prevalence of Medication Errors and the
}

\section{Associated Factors: A Prospective Observational Study Among Cancer Patients at Mbarara Regional Referral Hospital}

\author{
Abigaba Dorothy ${ }^{1,2}$ \\ Tadele Mekuriya Yadesa $\mathbb{D}^{1,2}$ \\ Esther Atukunda (D) \\ 'Department of Pharmacy, Mbarara \\ University of Science and Technology, \\ Mbarara, Uganda; ${ }^{2}$ World Bank, ACE II, \\ Pharmacy Biotechnology and Traditional \\ Medicine Center, Mbarara University of \\ Science and Technology, Mbarara, Uganda
}

Background: Medication error is one of the most common medical errors in the practice of modern medicine. Among cancer patients receiving chemotherapy, medication errors can be potentially harmful given the narrow therapeutic index, complex dosing, and toxic nature of anti-cancer drugs.

Objective: This study aimed to determine the incidence and factors associated with medication errors among cancer patients.

Methods: The study was a prospective observational study carried out at the cancer unit of Mbarara Regional Referral Hospital, Southwestern Uganda. The study included 110 participants, both adults and children receiving chemotherapy. The study was carried out for a period of five months from January to May 2020. A checklist was used to collect patient, medication, and disease information to identify the prescription, transcription, dispensing, and administration errors.

Results: Of the 110 participants, 52 (47.3\%) experienced a total of 78 medication errors (MEs). Of these, 33 (42.31\%) were prescription errors, 29 (37.18\%) administration errors, 9 (11.54\%) transcription errors, and 7 (8.97\%) dispensing errors. In the adjusted logistic regression of factors associated with medication errors, urban residents (aOR, 4.59; 95\% CI, 1.08, 19.53, $p=0.039$ ) and educated participants (at secondary level) (aOR, 10.51; 95\% CI, 1.43, 77.14, $p=0.021$ ) had a significantly higher risk of experiencing medication errors. Participants treated with alkylating agents (aOR, 2.87; 95\% CI, 1.07, 7.72, $p=0.036$ ) had a greater risk of experiencing medication errors when compared to other classes of chemotherapy.

Conclusion: The incidence of medication errors among cancer patients was high in Mbarara Regional Referral Hospital. Prescription errors were the most common type of error followed by administration errors, and dispensing errors were the least common. Residence, education level, and alkylating agent chemotherapy were significantly associated with occurrence of medication errors.

Keywords: prevalence, medication error, associated factors, cancer, Mbarara

\section{Introduction}

Medication error is one of the most common medical errors in the practice of modern medicine. ${ }^{1}$ According to (C) 2021 National Coordinating Council for Medication Error Reporting and Prevention, All Rights Reserved, medication errors are defined as:
Correspondence: Abigaba Dorothy Tel +256 775591958

Email abigabadorothy@gmail.com 
any preventable event that may cause or lead to inappropriate medication use or patient harm while the medication is in the control of the health care professional, patient, or consumer. Such events may be related to professional practice, health care products, procedures, and systems. ${ }^{1}$

There is an increasing incidence of both adult and pediatric cancer cases in developing countries, thus, there is equally a rise in the use of anti-cancer drugs as one of the cancer treatments. ${ }^{2}$ However, with the increase in drug use, there is also an increasing chance of medication errors which can occur at any stage of prescription, transcription, dispensing, and administration of anti-cancer drugs resulting in disability, prolonged hospitalization, and mortality. ${ }^{3}$ Chemotherapy-related medication errors can be potentially harmful given the narrow therapeutic index, complex dosing, and toxic nature of anticancer drugs especially among the already immunecompromised cancer patients. ${ }^{1}$ A review of data about medication errors among cancer patients indicated that $1-3 \%$ of patients experienced a medication error during treatment. ${ }^{4}$ In the USA, the incidence of medication errors among cancer patients has been reported at $7.1 \%$ among adults and $18.8 \%$ among pediatric patients. ${ }^{5}$

Concisely, medication errors impose great economic impact on the healthcare system, society, and patients. The impact on health organizations, health workers, and patients include prolonged hospitalization, loss of trust, and loss of productivity respectively. ${ }^{6}$

Data reviewed about medication errors in Africa showed that $13 \%$ to $76 \%$ of prescriptions for general patients had a medication error. ${ }^{7}$ A study in Nigeria revealed that prescription medication error was the most common medical error at $95.2 \%$ among medical personnel. ${ }^{8}$ In Uganda, $18 \%$ of health care professionals disclosed having made medication errors while treating patients. ${ }^{9}$ Medication errors are therefore a common occurrence among inpatient and outpatient encounters. There has been no similar research conducted in the study area on incidence of medication errors and specifically data about cancer-related medication errors. Accordingly, this study aimed to determine the incidence and factors associated with medication errors in a cancer unit in Uganda.

\section{Methods}

\section{Study Design and Setting}

The study was conducted at the cancer unit of Mbarara Regional Referral Hospital, Southwestern Uganda. The ward has a bed capacity of 20 beds for adults and 18 beds for pediatric cancer patients with two oncology specialists, one pharmacist, and seven nurses. The prospective observational study was carried out among patients on cycle one and two of treatment who were being treated at cancer unit of MRRH from January 2020 to May 2020.

\section{Study Participants}

All 110 patients who were receiving cycle one and two chemotherapy treatment at cancer unit of MRRH during the study period, who fulfilled the inclusion and exclusion criteria were studied.

\section{Inclusion Criteria}

The study included:

- Patients of all age groups.

- Patients with definitive diagnosis of cancer who were receiving chemotherapy or other adjuvant cancer drugs in cycle one and two of treatment.

- Patients who were able to provide a signed consent form (for adults) and consent obtained from caretakers of pediatric patients. Assent was also obtained from children and their care takers who were able to understand the study.

\section{Exclusion Criteria}

- Patients without a definitive cancer diagnosis and not receiving chemotherapy treatment did not participate in the study.

- Individuals that were unable to provide consent or assent were also excluded from the study.

\section{Data Collection}

A checklist was developed based on the American Society of Health-System Pharmacists ${ }^{10}$ classification of medication errors and used to document observed errors from each patient's medication while admitted to the cancer unit. The checklist included different drug prescription, transcription, dispensing, and administration errors.

Prescription errors were assessed by comparison of patient prescriptions with standard treatment protocol from Uganda Cancer Institute treatment guidelines and Physicians' Cancer Chemotherapy Drug Manual ${ }^{11}$ with anti-cancer drugs that determined whether the prescriptions fulfilled the five-rights of appropriate drug use and correct frequency with duration of treatment regimen. The five-rights of drug use include right drug for the right 
patient with the right indication at the right dose using the right route of administration. Transcription errors were determined from assessment of any differences in the patient prescription and drug transcription forms which are the basis for drug preparation.

Dispensing errors were identified as labeling errors, wrong drug, wrong doses, and wrong patients. Administration errors were differences in time of administration, frequency of administration, and route of administration. Following prescription for the study patient, the patient's prescription was assessed on whether the prescription followed the protocol for particular cancer treatment for the drug, calculated dose per body surface area, route, frequency, and duration prescribed.

After prescription the drugs are transcribed to the dispensing form that is used for preparation and dispensing of chemotherapy. The dispensing form was assessed for transcription error in comparison with the prescription. Following preparation, the drugs are labeled with patient name, drug name, dose as prescribed. Errors were identified based on the protocol and prescription. During drug administration after dispensing, the patient was observed for route, time, duration, and rate of administration. The errors were identified from the comparison with the treatment protocol.

To minimize the possible consequent bias, the errors identified prospectively were not corrected, except in few cases when investigators believed there was potentially life-threatening medication errors, when intervention was made during medical rounds without letting the health care workers know it was part of the study's finding.

Accuracy of the data collected was ensured through training of data collection assistants, close supervision of data collection process, and giving feedback in case of difficulties encountered. In addition, a pretest which involved five percent ( 9 patients) of the estimated sample size population was conducted and the feedback used to improve the data collection tools. The results obtained were not used in the final analysis of the study.

\section{Data Processing and Analysis}

The information obtained from individual patients' checklists was transferred to Excel. The collected data were analyzed using Stata version 12. Descriptive statistics were used to analyze frequency and percentage of medication errors among different variables. Chi-square d test was used to determine distribution and types of medication error among different categories of patients.
The association between each independent variable and medication error was analyzed using univariate logistic regression to obtain crude odds ratios. P-value less than 0.05 was considered significant. All variables with $\mathrm{P}<$ 0.25 were fit into a multivariate logistic regression model to control for confounders and obtain adjusted odds ratios.

\section{Ethical Considerations}

This study was conducted in accordance with the Declaration of Helsinki. ${ }^{12}$ The study was approved by MUST Research and Ethics Committee (REC) with an approval letter Ref: MUREC 1/7-2020. Consent was sought before recruitment of adult patients while parental consent and child assent was obtained for children below eighteen years.

\section{Results}

\section{Participant Characteristics}

Of the 110 participants enrolled in this study, $67(60.9 \%)$ were male, $34(30.9 \%)$ were above 65 years of age, 68 (61.8\%) were Munyankole ethnicity, 54 (49.1\%) were rural residents, $80(72.7 \%)$ participants had no comorbidities, 57 (51.8\%) of the cancers diagnosed were not staged, $47(42.7 \%)$ had not attained any formal education, and 66 $(60.0 \%)$ were underweight. More than half of the participants were diagnosed with carcinomas, 67 (60.9\%), while $63(57.3 \%)$ were treated using alkylating agents as chemotherapy (Table 1).

\section{Prevalence of Medication Errors}

Overall, $52(47.3 \%)$ participants had at least one medication error when all the four sub-categories of medication errors were considered (Figure 1).

\section{Types of Medication Errors}

The study showed that $33(42.31 \%)$ prescription errors were the most commonly occurring, followed by 29 (37.18\%) which were administration errors (Table 2). There were $9(11.54 \%)$ transcription errors and 7 $(8.97 \%)$ dispensing errors; the least common. Of the prescription errors, wrong dose prescribed (18) and wrong frequency (10) were the most common, while of the administration errors, wrong infusion rate (17) of administration was the most common. 
Table I Participant Characteristics $(n=\mid 10)$

\begin{tabular}{|c|c|c|}
\hline Characteristics & Level & Frequency (\%) \\
\hline \multirow{4}{*}{$\begin{array}{l}\text { Age category } \\
\text { (years) }\end{array}$} & $<18$ & $28(25.5)$ \\
\hline & $18-49$ & $27(24.5)$ \\
\hline & $50-64$ & $21(19.1)$ \\
\hline & $\geq 65$ & $34(30.9)$ \\
\hline \multirow[t]{2}{*}{ Sex } & Male & $67(60.9)$ \\
\hline & Female & $43(39.1)$ \\
\hline \multirow[t]{4}{*}{ Tribe } & Munyankole & $68(61.8)$ \\
\hline & Baganda & $7(6.4)$ \\
\hline & Mukiga & $21(19.1)$ \\
\hline & Others & $14(12.7)$ \\
\hline \multirow[t]{3}{*}{ Residence } & Urban & $18(16.4)$ \\
\hline & Peri-urban & $38(34.5)$ \\
\hline & Rural & $54(49.1)$ \\
\hline \multirow[t]{4}{*}{ Level of education } & None & $47(42.7)$ \\
\hline & Primary & $41(37.3)$ \\
\hline & Secondary & $10(9.1)$ \\
\hline & Tertiary & $12(10.9)$ \\
\hline \multirow{4}{*}{$\begin{array}{l}\text { Body mass index } \\
\text { (BMI) }\end{array}$} & Underweight & $66(60.0)$ \\
\hline & Normal weight & $26(23.6)$ \\
\hline & Overweight & $17(15.5)$ \\
\hline & Obese & I (0.9) \\
\hline \multirow[t]{5}{*}{ Comorbidity } & None & $80(72.7)$ \\
\hline & DM & $3(2.7)$ \\
\hline & Hypertension & $8(7.3)$ \\
\hline & HIV & $10(9.1)$ \\
\hline & Others & $9(8.2)$ \\
\hline \multirow[t]{4}{*}{ Stage of cancer } & $\|$ & $7(6.4)$ \\
\hline & III & $15(13.6)$ \\
\hline & IV & $31(28.2)$ \\
\hline & Not staged & $57(51.8)$ \\
\hline \multirow[t]{5}{*}{ Cancer diagnosis } & Carcinoma & $67(60.9)$ \\
\hline & Sarcoma & $15(13.6)$ \\
\hline & Leukemia & $9(8.2)$ \\
\hline & Lymphoma & $8(7.3)$ \\
\hline & Others & $10(9.1)$ \\
\hline
\end{tabular}

(Continued)
Table I (Continued).

\begin{tabular}{|l|l|l|}
\hline Characteristics & Level & Frequency (\%) \\
\hline $\begin{array}{l}\text { Chemotherapy } \\
\text { drugs }\end{array}$ & Alkylating agents & $63(57.3)$ \\
\cline { 2 - 3 } & Anti-metabolites & $25(22.7)$ \\
\cline { 2 - 3 } & Anthracyclines & $53(48.2)$ \\
\cline { 2 - 3 } & Topoisomerase inhibitors & $2(1.8)$ \\
\cline { 2 - 3 } & Plant alkaloid & $46(41.8)$ \\
\cline { 2 - 3 } & Corticosteroids & $14(12.8)$ \\
\cline { 2 - 3 } & Other treatments & $50(45.5)$ \\
\hline
\end{tabular}

\section{Bivariate Logistic Regression for Factors Associated with Medication Error}

In the unadjusted analysis, data showed that urban residents (OR, 3.69; 95\% CI, 1.15-11.79, $p=0.028$ ) were more likely to experience medication errors compared to rural and periurban residents. Among participants who attained education, participants with secondary level education (OR, 5.97; 95\% CI, 1.11-32.1, $p=0.037$ ) were more likely to experience medication errors. Participants receiving alkylating agents were also more likely to experience medication errors (OR 3.04; 95\% CI, 1.37-6.70, $p=0.006$ ) (Table 3).

\section{Multivariate Analysis of the Factors Associated with MEs}

Multivariate analysis showed that urban residents were more likely to experience medication errors [adjusted

\section{Prevalence of medication errors}

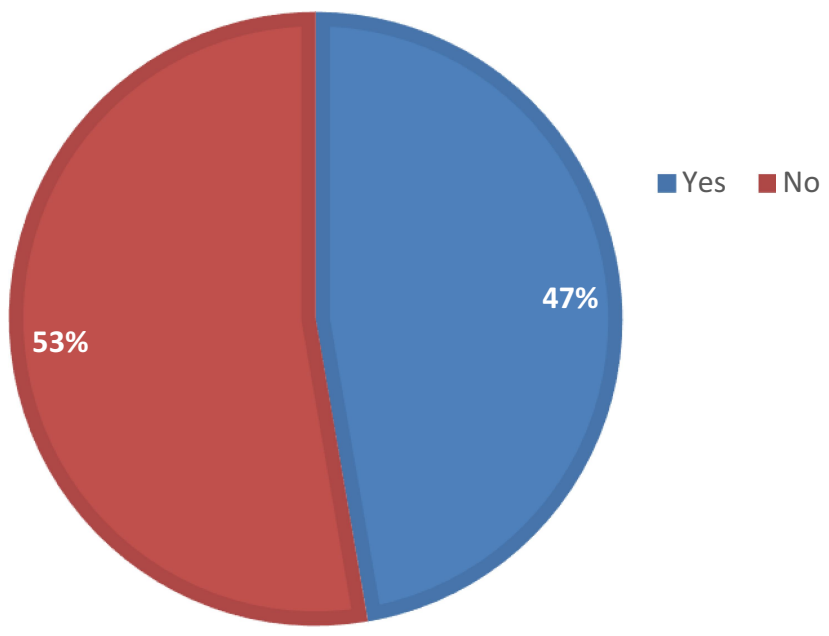

Figure I Prevalence of medication errors among cancer patients at MRRH. 
Table 2 Description of the Types and Categories of Medication Errors Observed Among Cancer Patients at Mbarara Regional Referral Hospital

\begin{tabular}{|l|l|}
\hline $\begin{array}{l}\text { Types of Medication Error } \\
\text { (Frequency) }\end{array}$ & Description (Frequency) \\
\hline Prescription errors (33) & Drug dose (18) \\
\cline { 2 - 2 } & Wrong frequency prescribed (10) \\
\cline { 2 - 2 } & Wrong route prescribed (2) \\
\cline { 2 - 2 } & Others (2) \\
\cline { 2 - 2 } & Wrong drug (1) \\
\hline \multirow{2}{*}{ Administration error (29) } & Wrong infusion rate (17) \\
\cline { 2 - 2 } & Others (12) \\
\hline \multirow{2}{*}{ Transcription error (9) } & Wrong dose (3) \\
\cline { 2 - 2 } & Wrong time (3) \\
\cline { 2 - 2 } & Others (3) \\
\hline \multirow{3}{*}{ Dispensing error (7) } & Wrong frequency (4) \\
\cline { 2 - 2 } & Wrong dose (3) \\
\hline
\end{tabular}

odds ratio (aOR), 4.59; 95\% CI, 1.08, 19.53, $p=0.039]$ compared to rural and peri-urban residents. Participants with secondary education were associated with more medication errors (aOR 10.51; 95\% CI, 1.43, 77.14, $p=0.021$ ). Compared to participants receiving any other type of chemotherapy, participants on alkylating agents were more likely to experience a medication error (aOR 2.87; $95 \%$ CI, 1.07, 7.72, $p=0.036$ ) (Table 4).

\section{Discussion}

We conducted a study with the aim of documenting the prevalence of medication errors and the associated factors among cancer inpatients and outpatients at Mbarara Regional Referral Hospital from January to May 2020. Results revealed that during that period, 78 medication errors occurred, at a prevalence of $47.3 \%$ and most commonly, prescription errors $(42.3 \%)$. The categories of wrong dose errors (54.5\%) and frequency errors (30.4\%) occurred more commonly. Administration errors were also frequent, unlike dispensing errors. Both bivariate and multivariate analyses showed that urban residents, participants who attained secondary level education, and those who received alkylating agents were more likely to experience medication errors.
Our results are in line with previous studies, for example; a study in South India documented a prevalence of $41.6 \%$, with prescription errors as the most common type of error. ${ }^{13}$ The most common error was dose-related with $79(22.83 \%)$ cases of overdosing. However, the current prevalence is much higher compared to the finding of a study in Brazil that reported a prevalence of $6.24 \%{ }^{14}$ as well as another study in France that documented prevalence at $5.2 \% .{ }^{15}$ These differences are probably because of the better health care systems and lower prescriber or nurse to patient ratio in middle income and high-income countries compared to our study that was conducted in a low-income country.

On the contrary, the current prevalence $(47.3 \%)$ is lower than that reported in Pakistan $(62 \%) .{ }^{16}$ This notable difference is probably due to the difference in methodology since the study included lack of effectiveness as one of the other errors involving drugs. The lower error prevalence observed in our study could also be due to human error since the prescription process is highly human dependent, characterized by hand written prescription and drug process orders without secondary verification procedures. These differences could also be attributed to the use of CPOE, prescription validation systems and training received by health care workers, a crucial component that is lacking in a developing country with poor healthcare systems.

Our study also revealed that prescription errors were the most common error type, a finding similar to previous studies conducted in the United States, ${ }^{17}$ Spain, ${ }^{18}$ and Brazil. ${ }^{14}$ The high frequency of errors during the prescription process could be due to the shortage of oncology specialists to supervise the prescription process. The absence of updated specific cancer treatment protocols for individual patient files, accompanied by inadequate continuous training of prescribers on efficient use of protocols, could also have led to increased error occurrence during prescriptions. This is because treatment protocols guide prescribers on choice of drug regimen during prescriptions, hence errors could arise from their incorrect use and unavailability. ${ }^{19}$

Administration errors contributed $37.2 \%$ of all medication errors, compared to previous studies that documented $32 \%$ and $39 \%$ in the United Kingdom and Ethiopia respectively. ${ }^{20,21}$ However, a study from Iran indicated $26.5 \%$, possibly due to the higher number of nursing staff involved in patient care compared to those in this study. ${ }^{22}$ Higher prevalence of administration errors was 
Table 3 Bivariate Logistic Regression Analysis of the Independent Factors with MEs

\begin{tabular}{|c|c|c|c|c|c|c|}
\hline \multicolumn{3}{|l|}{ Characteristics } & \multicolumn{4}{|c|}{ Medication Errors } \\
\hline & \multirow{2}{*}{\multicolumn{2}{|c|}{ Level }} & No $(n=58)$ & Yes $(n=52)$ & Unadjusted OR $(95 \% \mathrm{Cl})$ & P-value \\
\hline & & & No. (\%) & No. (\%) & & \\
\hline \multirow[t]{4}{*}{ Age category (years) } & \multicolumn{2}{|l|}{$<18$} & $16(27.6)$ & $12(23.1)$ & 1 & \\
\hline & \multicolumn{2}{|l|}{$18-49$} & $14(24.1)$ & $13(25)$ & $1.24(0.43,3.58)$ & 0.694 \\
\hline & \multicolumn{2}{|l|}{$50-64$} & $9(15.5)$ & $12(23.1)$ & $1.78(0.57,5.58)$ & 0.324 \\
\hline & \multicolumn{2}{|l|}{$\geq 65$} & $19(32.76)$ & $15(28.9)$ & $1.05(0.35,1.58)$ & 0.921 \\
\hline \multirow[t]{2}{*}{ Sex } & \multicolumn{2}{|l|}{ Female } & $23(41.4)$ & $20(36.5)$ & 1 & \\
\hline & \multicolumn{2}{|l|}{ Male } & $35(60.3)$ & $32(61.5)$ & $1.05(0.48,2.26)$ & 0.898 \\
\hline \multirow[t]{3}{*}{ Residence } & \multicolumn{2}{|l|}{ Rural } & $29(50.0)$ & $22(42.3)$ & 1 & \\
\hline & \multicolumn{2}{|l|}{ Peri-urban } & $24(41.4)$ & $16(30.8)$ & $0.88(0.38,2.04)$ & 0.763 \\
\hline & \multicolumn{2}{|l|}{ Urban } & $5(8.6)$ & $14(26.9)$ & $3.69(1.15,11.79)$ & 0.028 \\
\hline \multirow[t]{4}{*}{ Level of education } & \multicolumn{2}{|l|}{ None } & $29(50.0)$ & $17(32.7)$ & 1 & \\
\hline & \multicolumn{2}{|l|}{ Primary } & $22(37.9)$ & $21(40.4)$ & $1.62(0.69,3.8)$ & 0.259 \\
\hline & \multicolumn{2}{|l|}{ Secondary } & $2(3.5)$ & $7(13.5)$ & $5.97(1.11,32.1)$ & 0.037 \\
\hline & \multicolumn{2}{|l|}{ Tertiary } & $5(8.6)$ & $7(13.5)$ & $2.39(0.65,8.7)$ & 0.187 \\
\hline \multirow[t]{5}{*}{ Comorbidity } & \multicolumn{2}{|l|}{ None } & $43(74.1)$ & $37(7 \mid .2)$ & 1 & \\
\hline & \multicolumn{2}{|l|}{ DM } & $\mathrm{I}(\mathrm{I} .7)$ & I (I.9) & $1.16(0.07,19.23)$ & 0.916 \\
\hline & \multicolumn{2}{|l|}{ Hypertension } & $6(10.3)$ & $3(5.8)$ & $0.58(0.14,2.49)$ & 0.464 \\
\hline & \multicolumn{2}{|l|}{ HIV } & $5(8.6)$ & $5(9.6)$ & $1.16(0.31,4.32)$ & 0.823 \\
\hline & \multicolumn{2}{|l|}{ Others } & $2(3.4)$ & $7(13.5)$ & $2.32(0.54,9.94)$ & 0.256 \\
\hline \multirow[t]{4}{*}{ Stage of cancer } & \multicolumn{2}{|l|}{ II } & $5(8.6)$ & $2(3.8)$ & 1 & \\
\hline & III & & $9(15.5)$ & $6(11.5)$ & $2.5(0.36,17.5)$ & 0.356 \\
\hline & IV & & $10(17.2)$ & $21(40.4)$ & $4.55(0.75,27.42)$ & 0.099 \\
\hline & Not staged & & $34(58.6)$ & $23(44.2)$ & $1.64(0.29,9.19)$ & 0.572 \\
\hline Cancer diagnosis & Carcinoma & No & $25(43.1)$ & $18(34.6)$ & 1 & \\
\hline & & Yes & $33(56.9)$ & $34(65.4)$ & $1.43(0.66,3.10)$ & 0.363 \\
\hline & Sarcoma & No & $5 \mathrm{I}(87.9)$ & $44(84.6)$ & 1 & \\
\hline & & Yes & $7(12.1)$ & $8(15.4)$ & $1.32(0.44,3.95)$ & 0.614 \\
\hline & Leukemia & No & $50(86.2)$ & $50(96.2)$ & 1 & \\
\hline & & Yes & $8(13.8)$ & $2(3.9)$ & $0.25(0.05,1.24)$ & 0.089 \\
\hline & Lymphoma & No & $55(94.8)$ & $47(90.4)$ & 1 & \\
\hline & & Yes & $3(5.2)$ & $5(9.6)$ & $1.95(0.44,8.6)$ & 0.377 \\
\hline & Others & No & $5 \mathrm{I}(87.9)$ & $49(94.2)$ & 1 & \\
\hline & & Yes & $7(12.1)$ & $3(5.8)$ & $0.45(0.11,1.82)$ & 0.261 \\
\hline
\end{tabular}

(Continued) 
Table 3 (Continued).

\begin{tabular}{|c|c|c|c|c|c|c|}
\hline \multicolumn{3}{|c|}{ Characteristics } & \multicolumn{4}{|c|}{ Medication Errors } \\
\hline & \multirow{2}{*}{\multicolumn{2}{|c|}{ Level }} & No $(n=58)$ & Yes $(n=52)$ & Unadjusted OR ( $95 \% \mathrm{Cl})$ & P-value \\
\hline & & & No. (\%) & No. (\%) & & \\
\hline \multirow{14}{*}{$\begin{array}{l}\text { Chemotherapy } \\
\text { drugs }\end{array}$} & \multirow[t]{2}{*}{ Alkylating agents } & No & $32(55.2)$ & $15(28.9)$ & 1 & \\
\hline & & Yes & $26(44.8)$ & $37(71.2)$ & $3.04(1.37,6.70)$ & 0.006 \\
\hline & \multirow[t]{2}{*}{ Anti-metabolites } & No & $44(75.9)$ & $4 \mid(78.9)$ & 1 & \\
\hline & & Yes & $14(24.1)$ & $\mathrm{II}(2 \mid .2)$ & $0.84(0.34,2.07)$ & 0.709 \\
\hline & \multirow[t]{2}{*}{ Anthracyclines } & No & $28(48.3)$ & $29(55.8)$ & 1 & \\
\hline & & Yes & $30(51.7)$ & $23(44.2)$ & $0.74(0.34,1.6)$ & 0.433 \\
\hline & \multirow{2}{*}{$\begin{array}{l}\text { Topoisomerase } \\
\text { inhibitors }\end{array}$} & No & $57(98.3)$ & $51(98.1)$ & 1 & \\
\hline & & Yes & $\mathrm{I}(\mathrm{I} .7)$ & $\mathrm{I}(1.92)$ & $1.12(0.07,18.3)$ & 0.938 \\
\hline & \multirow[t]{2}{*}{ Plant alkaloid } & No & $34(58.6)$ & $30(57.7)$ & 1 & \\
\hline & & Yes & $24(41.4)$ & $22(42.3)$ & $1.03(0.48,2.2)$ & 0.921 \\
\hline & \multirow[t]{2}{*}{ Corticosteroids } & No & $51(87.9)$ & $45(86.5)$ & 1 & \\
\hline & & Yes & $7(12.1)$ & $7(13.5)$ & $1.13(0.36,3.5)$ & 0.827 \\
\hline & \multirow[t]{2}{*}{ Other treatments } & No & $29(50)$ & $31(59.6)$ & 1 & \\
\hline & & Yes & $29(50.0)$ & $21(40.4)$ & $0.68(0.32,1.4)$ & 0.313 \\
\hline
\end{tabular}

registered in a study conducted in United States $(56 \%),{ }^{5}$ probably due to the inclusion of preparation errors under the administration process which differs from our study. Rate of infusion errors $(58.6 \%)$ were the most common in this category under drug administration. This prevalence is higher than that reported by a study in Turkey which had an outcome of $39.7 \%^{2}$ and $35.2 \%$ from Iran. ${ }^{22}$ This finding could be attributed to the use of infusion pumps that are

Table 4 Multivariate Logistic Regression Analysis of the Independent Factors with MEs

\begin{tabular}{|c|c|c|c|c|}
\hline \multirow[t]{2}{*}{ Characteristics } & \multirow{2}{*}{\multicolumn{2}{|c|}{ Level }} & \multicolumn{2}{|c|}{ Adjusted Odds Ratio } \\
\hline & & & aOR $(95 \% \mathrm{Cl})$ & P-value \\
\hline \multirow[t]{3}{*}{ Residence } & \multicolumn{2}{|l|}{ Rural } & Ref & \\
\hline & \multicolumn{2}{|l|}{ Peri-urban } & $0.89(0.34,2.32)$ & 0.811 \\
\hline & \multicolumn{2}{|l|}{ Urban } & $4.59(1.08,19.53)$ & 0.039 \\
\hline \multirow[t]{4}{*}{ Level of education } & \multicolumn{2}{|l|}{ None } & Ref & \\
\hline & \multicolumn{2}{|l|}{ Primary } & I.54 $(0.59,4.02)$ & 0.376 \\
\hline & \multicolumn{2}{|l|}{ Secondary } & $10.51(1.43,77.14)$ & 0.021 \\
\hline & \multicolumn{2}{|l|}{ Tertiary } & $1.37(0.24,7.6)$ & 0.719 \\
\hline \multirow[t]{2}{*}{ Chemotherapy drugs } & \multirow[t]{2}{*}{ Alkylating agents } & No & Ref & \\
\hline & & Yes & $2.87(1.07,7.72)$ & 0.036 \\
\hline
\end{tabular}

Notes: I) Adjusted odds ratios were expressed using exponentiated coefficients; $95 \%$ confidence intervals in brackets; 2) bolded figures indicate statistically significant associations.

Abbreviations: $O R$, unadjusted odds ratio; aOR, adjusted odds ratio. 
necessary for standardizing drug delivery rates, which are absent in our study setting.

The other category of errors was under transcription with $11.54 \%$ contribution to the total errors. Wrong frequency transcribed, wrong doses, and other transcription errors contributed $33.3 \%$. The total error is lower than the $21 \%$ reported by Ford et al and Schwappach and Wernli. $^{23,24}$ The observed transcription errors can be attributed to multitasking required by the few pharmacy staff required to transcribe, prepare, and dispense chemotherapy. In addition, WHO links such errors to poor handwriting and lack of communication between health workers. $^{25}$

Dispensing errors $(8.97 \%)$ were the least common as the drugs are mostly dispensed by trained pharmacists that frequently double-check before dispensing which reduces the risk of errors. ${ }^{16}$ The minor occurrences can be attributed to workload, and long work shifts since the pharmacist to patient ratio is very low.

Among the factors identified to be related to occurrence of medication error was urban residence, ie, urban residents experienced more medication errors compared to those in semi-urban and rural areas. This could be explained by an observation made that people living in urban areas preferred to be outpatients which compelled them to alter the drug infusion rate in order to leave hospital on the same day hence reducing the monitoring given to these patients compared to the inpatients.

In addition to residence, more errors were reported among the educated participants compared to the uneducated, with the greatest risk being among participants who had attained education at secondary school level. This is possibly linked to the settlement areas, ie, the educated are commonly found in urban areas. However, this is contrary to most studies which have revealed that more errors have been observed among the uneducated in studies carried out in community pharmacies due to inability to understand instructions and comprehend health education. ${ }^{26}$

Participants who were treated with alkylating agents experienced more medication errors compared to other classes of chemotherapy treatment. This differs from the observation made in a study conducted among the pediatric population where anti metabolites were more associated with medication errors at $39.5 \%$ and alkylating agents at $14 \%{ }^{27}$ However, WHO links errors associated with alkylating agents to the necessity to alter doses which is often not done as required. ${ }^{28}$
The strengths of this study include prospective data collection which captured errors that could have been missed by retrospective method and using the patient study population which provided less biased information on occurrence of errors compared to health workers. However, some of the study limitations include a short study period of only five months, small pediatric study population, preparation process stage was not considered, and exclusion of documentation error under administration process.

\section{Conclusion}

Almost half of the patients at Mbarara Regional Referral Hospital experienced at least one medication error. The prevalence was comparable to other previous studies from developing countries. Prescription errors were the most common type of error which is comparable to other previous studies; mainly due to wrong dose being prescribed. Administration errors were second most frequent which was mostly due to wrong rate of infusion. Residence, education level, and alkylating agents used as chemotherapy were significantly associated with occurrence of medication errors.

Prescribers should take extra precautions when they prescribe chemotherapy by adhering to treatment protocols. Additionally, CPOE should be adopted to reduce human error related to hand-written prescriptions. Hospitals should avail chemotherapy infusion pumps to reduce errors resulting from rate of infusion. Patient health education is highly recommended in order to prevent medication errors caused by patients' lack of knowledge regarding complexity of their treatment.

\section{Abbreviations}

aOR, adjusted odds ratio; OR, odds ratio; DM, diabetes mellitus; HTN, hypertension; HIV, human immunodeficiency virus; CPOE, computerized physician order entry; MRRH, Mbarara University of Science and Technology; WHO, World Health Organization.

\section{Data Sharing Statement}

All the data supporting the conclusions of this article are included within this manuscript.

\section{Ethics Approval and Consent to Participate}

The study protocol was approved by the Research Ethics Committee (REC) at Mbarara University of Science and Technology as the Institutional review board for 
authorization to conduct the research (MUREC 1/7-2020). Informed consent was sought before recruitment of adult patients while parental or guardian consent was obtained on behalf of children below 18 years. All methods used in the study were in accordance with regulations and guidelines of the Research Ethics Committee and cancer unit of Mbarara Regional Referral Hospital.

\section{Consent for Publication}

All authors agreed to submission of this manuscript for publication in addition to the consent to publish which was included in the informed consent form which attained ethical and participant approval.

\section{Acknowledgments}

The authors extend their sincere gratitude toward the study participants and health workers at the cancer unit of Mbarara Regional Referral Hospital. We would like to thank the data collection assistants for cooperating with us through the data collection process.

\section{Author Contributions}

All authors made a significant contribution to the work reported, whether that is in the conception, study design, execution, acquisition of data, analysis and interpretation, or in all these areas; took part in drafting, revising or critically reviewing the article; gave final approval of the version to be published; have agreed on the journal to which the article has been submitted; and agree to be accountable for all aspects of the work.

\section{Funding}

This study was funded by PHARMBIOTRAC a project under the funding of World Bank at Mbarara University of Science and Technology.

\section{Disclosure}

The authors declare that they have no conflicts of interest for this work.

\section{References}

1. Mattsson T, Holm B, Michelsen H, Knudsen J, Brixen K, Herrstedt J. Non-intercepted dose errors in prescribing anti-neoplastic treatment: a prospective, comparative cohort study. Ann Oncol. 2015;26 (5):981-986. doi:10.1093/annonc/mdv032

2. Ulas A, Silay K, Akinci S, et al. Medication errors in chemotherapy preparation and administration: a survey conducted among oncology nurses in Turkey. Asian Pac J Cancer Prev. 2015;16(5):1699-1705. doi:10.7314/APJCP.2015.16.5.1699
3. Krzyzaniak N, Bajorek B. Medication safety in neonatal care: a review of medication errors among neonates. Ther Adv Drug Saf. 2016;7(3):102-119. doi:10.1177/2042098616642231

4. Weingart SN, Zhang L, Sweeney M, Hassett M. Chemotherapy medication errors. Lancet Oncol. 2018;19(4):e191-e9. doi:10.1016/ S1470-2045(18)30094-9

5. Walsh KE, Dodd KS, Seetharaman K, et al. Medication errors among adults and children with cancer in the outpatient setting. $J$ Clin Oncol. 2009;27(6):891-896. doi:10.1200/JCO.2008.18.6072

6. Katongole SP, Anguyo RD, Nanyingi M, Nakiwala SR. Common medical errors and error reporting systems in selected Hospitals of Central Uganda. 2015.

7. Mekonnen AB, Alhawassi TM, McLachlan AJ, Brien J-AE. Adverse drug events and medication errors in African hospitals: a systematic review. Drugs Real World Outcomes. 2018;5(1):1-24. doi:10.1007/ s40801-017-0125-6

8. Iloh GUP, Chuku A, Amadi AN. Medical errors in Nigeria: a cross-sectional study of medical practitioners in Abia State. Arch Med Health Sci. 2017;5(1):44.

9. Kiguba R, Waako P, Ndagije HB, Karamagi C. Medication error disclosure and attitudes to reporting by healthcare professionals in a sub-Saharan African setting: a survey in Uganda. Drugs Real World Outcomes. 2015;2(3):273-287. doi:10.1007/s40801-015-0032-7

10. ASHP. ASHP guidelines on preventing medication errors in hospitals. Am J Hosp Pharm. 1993;50(2):305-314.

11. Edward C, De Vita VT Jr. Physicians' cancer chemotherapy drug manual 2015. Back to cited text; 2015:1.

12. World Medical Association. WMA Declaration of Helsinki - ethical principles for medical research involving human subjects 2018; [cited December 20, 2020]. Available from: https://www.wma.net/policiespost/wma-declaration-of-helsinki-ethical-principles-for-medicalresearch-involving-human-subjects/. Accessed April 13, 2021.

13. Mathaiyan J, Jain T, Dubashi B, Batmanabane G. Prescription, transcription and administration errors in out-patient day care unit of a regional cancer centre in South India. Asian Pac J Cancer Prev. 2016;17(5):2611-2617.

14. Duarte NC, Barbosa CR, Tavares MGR, Dias LP, Souza RN, Moriel P. Clinical oncology pharmacist: effective contribution to patient safety. $J$ Oncol Pharm Pract. 2018;25(7):1665-1674. doi:10.1177/1078155218807748

15. Ranchon F, Salles G, Späth H-M, et al. Chemotherapeutic errors in hospitalised cancer patients: attributable damage and extra costs. BMC Cancer. 2011;11(1):478. doi:10.1186/1471-2407-11-478

16. Azim M, Khan A, Khan TM, Kamran M. A cross-sectional study: medication safety among cancer in-patients in tertiary care hospitals in KPK, Pakistan. BMC Health Serv Res. 2019;19(1):583. doi:10.1186/s12913-019-4420-7

17. Ashokkumar R, Srinivasamurthy S, Kelly J, Howard S, Parasuraman S, Uppugunduri Satyanarayana CR. Frequency of chemotherapy medication errors: a systematic review. $J$ Pharmacol Pharmacother. 2018;9(2):86-91. doi:10.4103/jpp.JPP_61_18

18. Serrano-Fabiá A, Albert-Marí A, Almenar-Cubells D, Víctor Jiménez-Torres N. Multidisciplinary system for detecting medication errors in antineoplastic chemotherapy. J Oncol Pharm Pract. 2010;16 (2):105-112. doi:10.1177/1078155209340482

19. Goldspiel B, Hoffman JM, Griffith NL, et al. ASHP guidelines on preventing medication errors with chemotherapy and biotherapy. $\mathrm{Am}$ $J$ Health Syst Pharm. 2015;72(8):e6-e35. doi:10.2146/sp150001

20. Sutherland A, Canobbio M, Clarke J, Randall M, Skelland T, Weston E. Incidence and prevalence of intravenous medication errors in the UK: a systematic review. Eur J Hosp Pharm. 2020;27(1):3-8. doi:10.1136/ejhpharm-2018-001624

21. Gessese YA, Fenta TG, Weldegiorgis MA. Assessment of medication use process in adult oncology unit of Tikur Anbesa Specialized Hospital: a cross-sectional study in Addis Ababa, Ethiopia. Eur J Oncol Pharm. 2018;1(3):e0005. doi:10.1097/OP9.0000000000000005 
22. Tavakoli-Ardakani M, Omidi S, Eshraghi A, Salamzadeh J. Medication errors in administration of chemotherapeutic agents: an observational study. Iran J Pharm Sci. 2013;9(2):1-11.

23. Ford CD, Killebrew J, Fugitt P, Jacobsen J, Prystas EM. Study of medication errors on a community hospital oncology ward. J Oncol Pract. 2006;2(4):149-154. doi:10.1200/jop.2006.2.4.149

24. Schwappach D, Wernli M. Medication errors in chemotherapy: incidence, types and involvement of patients in prevention. A review of the literature. Eur J Cancer Care (Engl). 2010;19(3):285-292. doi:10.1111/j.1365-2354.2009.01127.x

25. World Health Organization. Global patient safety challenge: medication without Harm. Geneva: World Health Organization; 2017.
26. Sears K, Beigi P, Niyyati SS, Egan R. Patient-related risk factors for the occurrence of patient-reported medication errors in one community pharmacy: a local perspective. J Pharm Technol. 2016;32(1):3-8. doi: $10.1177 / 8755122515596539$

27. Rinke ML, Shore AD, Morlock L, Hicks RW, Miller MR. Characteristics of pediatric chemotherapy medication errors in a national error reporting database. Cancer. 2007;110(1):186-195. doi:10.1002/cncr.22742

28. World Health Organization. Medication safety in high risk situations; 2019.

\section{Publish your work in this journal}

Cancer Management and Research is an international, peer-reviewed open access journal focusing on cancer research and the optimal use of preventative and integrated treatment interventions to achieve improved outcomes, enhanced survival and quality of life for the cancer patient.
The manuscript management system is completely online and includes a very quick and fair peer-review system, which is all easy to use. Visit http://www.dovepress.com/testimonials.php to read real quotes from published authors. 